\title{
The effect of high intensity physical exercise and hypoxia on glycemia, angiogenic biomarkers and cardiorespiratory function in patients with type 1 diabetes
}

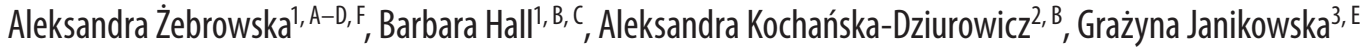 \\ ${ }^{1}$ Department of Physiological and Medical Sciences, Academy of Physical Education, Katowice, Poland \\ ${ }^{2}$ Department of Health Care, Silesian Medical College, Katowice, Poland \\ ${ }^{3}$ Department of Analytical Chemistry, Medical University of Silesia, Katowice, Poland \\ A - research concept and design; $B$ - collection and/or assembly of data; $C$ - data analysis and interpretation; \\ $D$ - writing the article; $E$ - critical revision of the article; $F$ - final approval of the article
}

\section{Address for correspondence}

Aleksandra Żebrowska

E-mail:a.zebrowska@awf.katowice.pl

\section{Funding sources}

None declared

Conflict of interest

None declared

\section{Acknowledgements}

We appreciate the help from physicians and nurses during the data collection period. In addition, we are grateful to those who participated in the study.

Received on June 17, 2016

Reviewed on July 19, 2016

Accepted on 0ctober 25, 2016

\begin{abstract}
Background. An integral part of the treatment of diabetes is physical activity. Scientific reports have shown the beneficial effects of hypoxia and exercise on cardiovascular and metabolic variables in patients with diabetes.
\end{abstract}

Objectives. The aim of the study was to assess the effect of normobaric hypoxia and exercise on the serum concentrations of proangiogenic factors and glycemia in patients with type 1 diabetes.

Material and methods. A total of 28 adults (aged 30.4 years \pm 9.7 years), suffering from diabetes for 12.1 years \pm 6.0 years and healthy individuals, participated in the following trials: normoxic (Nor) and hypoxic (Hy) rest and Nor and Hy incremental exercise test (Ex) $\left(\mathrm{FlO}{ }_{2}=15.2 \%\right)$. The Altitude Trainer Hypoxico System (HYP-123 Hypoxic Generator, LOWOXYGEN Technology GmbH, Berlin, Germany) corresponding to a height of about $2500 \mathrm{~m}$ above sea level was used in the study. Exercise tests were performed on a cycle ergometer Excalibur Sport (Lode B.V., Groningen, The Netherlands). Cardiorespiratory variables, glycemia, angiogenic and hematological indices were measured at rest and in response to both exercise protocols.

Results. The present data confirmed that the patients with type 1 diabetes demonstrated a good level of aerobic capacity and fitness. NorEx and HyEx resulted in a significant decrease in serum glucose concentration ( $p<0.05$ vs $p<0.01$ ). Patients with diabetes had higher baseline hypoxia induced factor-1alpha levels compared to healthy adults $(p<0.05)$, which increased after exposure to hypoxia and hypoxia with exercise $(p<0.001)$. Hypoxia significantly decreased baseline transforming growth factor- $\beta($ TGF- $\beta)(p<0.05)$ and had a significant effect on tumor necrosis factor-a level (TNF- $a)(F=4.9 ; p<0.05)$.

Conclusions. Our study demonstrated that hypoxia combined with exercise reduces glycemia and may induce significant benefits in the prevention of diabetes cardiovascular complications.

Key words: angiogenesis, type 1 diabetes mellitus, exercise tolerance, hypoxia-inducible factor-1

DOI

$10.17219 /$ acem $/ 66354$

Copyright

Copyright by Author(s)

This is an article distributed under the terms of the

Creative Commons Attribution Non-Commercial License

(http://creativecommons.org/licenses/by-nc-nd/4.0/) 


\section{Introduction}

Type 1 diabetes (T1DM) is an autoimmune disease resulting in the targeted destruction of pancreatic $\beta$-cells and permanent loss of insulin production. Currently, there is no clear evidence of the benefits of regular physical activity on glucose control in type 1 diabetes. However, regular exercise decreases the risk of diabetes related complications and ameliorates quality of life. ${ }^{1-3}$ It is widely accepted that individuals who have been using intensive insulin therapy can practice almost all types of physical activity. ${ }^{3,4}$ Recent studies suggest that an increasing number of individuals with type 1 diabetes are also practicing extreme sports (e.g., mountain climbing), even in high altitude conditions. ${ }^{5,6}$

Regular exercise increases glucose metabolism through an insulin-independent pathway, leads to increasing muscle oxidative capacity and constitutes the most effective stimulus for improvement in cardiovascular control. ${ }^{7-10}$ However, in people with diabetes, it may also be responsible for the occurrence of some adverse reactions, such as hypoglycemia, hyperglycemia, ketosis and diabetes related complications. The metabolic and cardiovascular side effects of exercise may depend on the starting levels of glycemia, type and/or intensity of exercise, and use of exogenous insulin. During intense exercise (> 85\% of maximal oxygen consumption - $\mathrm{VO}_{2 \max }$ ), the epinephrine response can augment hepatic glucose output and may lead to post exercise hyperglycemia. These can be mitigated by administrating short-acting insulin analogues before high intensity exercise. Prolonged exercise at moderate intensity $\left(40-60 \%\right.$ of $\left.\mathrm{VO}_{2 \max }\right)$ activates lipolysis, glycogenolysis and gluconeogenesis to allow an adequate supply of substrates for oxidative phosphorylation in contracting muscles, which exposes the patient to the risk of post-exercise hypoglycemia. ${ }^{2-4}$

Scientific reports have shown that hyperglycemia is mainly responsible for the development of micro- and/or macroangiopathy. ${ }^{11,12}$ Chronically elevated blood glucose concentration induces endothelium dysfunction, manifested mainly by impaired vascular dilation. Impaired vascular dilation, as well as hyperglycemia-induced disorders of platelet function, cause greater monocyte chemotactic activity and inflammation initiating the development of vascular complications. ${ }^{13}$ Diabetes related angiopathy can be aggravated by angiogenesis, which is mainly stimulated by hypoxia and more specifically by hypoxia-inducible factor-1 alpha (HIF-1 $\alpha$ ). Hypoxia increases the level of HIF-1 $\alpha$, which activates a number of genes related to hypoxia and stimulates angiogenic molecules such as vascular endothelial growth factor (VEGF), fibroblast growth factor (FGF), transforming growth factor beta (TGF- $\beta$ ), transforming growth factor alpha (TGF- $\alpha)$, and platelet-derived growth factor (PDGF). ${ }^{13,14}$

In diabetes, the chronic exposure of blood cells to hyperglycemia modifies the function of neutrophils which lose diapedesis ability. They plug the lumen of the vessel, leading to local hypoxia and potentially to angiogenesis intensification. ${ }^{14}$

Contrary to these observations, Mackenzie et al. support the findings that acute hypoxia and physical exercise appear to improve some aspects of glycemic control and might be a valuable therapeutic method in the treatment of people with type 2 diabetes. ${ }^{15}$ Exposure to hypoxia and muscle contractions improved short-term glycemic control, and decreased pro-inflammatory cytokines and insulin resistance. ${ }^{16}$ It has been hypothesized that the effects of hypoxia may induce inflammatory responses indispensable for the ischemia-related neovascularization process in physically active patients with diabetes. ${ }^{14}$

Type 1 diabetes is associated with reduced skeletal muscle capillarization and deregulation of complex angiogenesis pathways. ${ }^{11,17,18}$ The severity of microvascular complications is associated with an increase in VEGF concentrations, which is the main angiogenic factor in poorly controlled patients. Chronic hyperglycemia may be responsible for the intensification of angiogenesis in the arterial walls, which can lead to atherosclerosis. ${ }^{19,20}$ The significance of other factors stimulating the synthesis of serum VEGF and VEGF-A mRNA expression, such as cytokines, insulin-like growth factor-1 (IGF-1), nitric oxide, glycation end products, and reactive oxygen species, has also been reported. ${ }^{12,16,20}$

The physiological responses to lowered inspired oxygen pressure, either mediated by barometric pressure reduction (hypobaric hypoxia) or by lowering the oxygen fraction (normobaric hypoxia), in diabetes mellitus are, however, controversial. ${ }^{10,16,21,22}$ Hence, we aimed to evaluate the effect of normobaric hypoxia with and without physical exercise on the serum concentrations of proangiogenic factors, glycemic control, and cardiorespiratory adaptation in patients with type 1 diabetes mellitus.

\section{Material and methods}

\section{Subjects}

Fourteen patients suffering from type 1 diabetes (T1DM) were randomly assigned to the study. All subjects were recruited at the Diabetes Clinic of the Silesian Center in Poland. Mean duration of diabetes was 12.1 (SD 6.0) years, and glycated hemoglobin $\left(\mathrm{HbA}_{1 \mathrm{c}}\right)$ was $55.2(7.8) \mathrm{mmol} / \mathrm{mol}$ (Table 1). All individuals with T1DM adhered to intensive insulin therapy (NovoRapid - Novo Nordisk, Denmark; Lantus - Lantus SoloStar, Sanofi-Aventis GmbH, Frankfurt, Germany, or Humalog - Humalog Eli Lilly, Houten, Nederland) and performed self-monitoring of blood glucose on glycemic control. Half of the patients were using continuous subcutaneous insulin infusion while the other half used multiple insulin injections. Patients were advised to reduce their basal insulin infusion by $50 \%$ prior to each physical test. 
Only patients free of diabetic complications were enrolled. The other criteria for inclusion were no personal history of other cardiovascular or metabolic diseases, no simultaneous participation in another trial, being free of any acute infections up to 1 week prior to the study, nonsmokers and with good exercise tolerance confirmed by the direct measurement of $\mathrm{VO}_{2 \max }$, and $\mathrm{HbA}_{1 \mathrm{c}}<8.0 \%$. The medical history and information about diabetes etiology of the study participants were prepared by medical personnel of the Silesian Center. The control group consisted of 14 healthy nonsmoking individuals (CG) of a similar age and aerobic efficacy.

A few days before the examination, the subjects were asked to abstain from exercise, and also from the consumption of alcohol and caffeinated drinks. For the entire duration of experiment, their diet, fasting glycemia and insulin dosage were monitored. Composition of the diet was calculated with a dedicated software (Dietus, B.U.I. InFit, Warszawa, Poland).

The study protocol was approved by the Local Ethics Committee and conformed to the standards set by the Declaration of Helsinki.

\section{Testing protocol}

Throughout the experiment, the patients and healthy control individuals participated in the following trials: normoxic (NorRest: $\mathrm{FIO}_{2}=20.9 \% ; \mathrm{P}=990 \mathrm{hPa}$ ) and hypoxic rest (HyRest: $\mathrm{FIO}_{2}=15.2 \% ; \mathrm{P}=990 \mathrm{hPa}$ ) and normoxic (NorEx: $\mathrm{FIO}_{2}=20.9 \%$; $=990 \mathrm{hPa}$ ) and hypoxic (HyEx: $\mathrm{FIO}_{2}=15.2 \% ; \mathrm{P}=990 \mathrm{hPa}$ ) graded exercise test. The protocol was based on 3 laboratory studies (ambient conditions: $21^{\circ} \mathrm{C}, 60 \%$ relative humidity) after an overnight fast and all studies were separated by at least 7 days. The participants body mass and composition were determined by means of Bioelectrical Impedance Analysis (BIA) (InBody 220 Data Management System, Biospace, Seoul, Korea).

The Altitude Trainer Hypoxico System (HYP-123 Hypoxic Generator, LOWOXYGEN Technology GmbH, Berlin, Germany). corresponding to a height of about $2500 \mathrm{~m}$ above sea level was used in the study.

\section{Normoxic and hypoxic rest}

In the first part of the experiment, the patients and healthy control individuals rested for 40 min under Nor and Hy conditions. Physiological variables and biochemical variables were measured immediately before the test (PreEx) and after 40 min of rest (NorRest and HyRest).

\section{Normoxic and hypoxic exercise test}

All subjects participated in 2 graded exercise tests performed on a cycle ergometer Excalibur Sport (Lode B.V., Groningen, The Netherlands) in normoxic (NorEx) and hypoxic (HyEx) conditions. The tests started with a 3-min warm-up; the intensity was then increased by $30 \mathrm{~W}$ every 3 min up to maximal exercise intensity. Pulmonary ventilation (VE), oxygen uptake $\left(\mathrm{VO}_{2}\right)$, and carbon dioxide output $\left(\mathrm{CO}_{2}\right)$ were measured continuously from the $6^{\text {th }}$ min prior to exercising and throughout each stage of the exercise load under both Nor and Hy conditions (Ergospirometr Metalyzer 3B-2R; Cortex Biophysik GmbH, Leipzig, Germany).

Blood response measures and biochemical variables were measured immediately before (PreEx) and at maximal exercise intensity (NorEx; HyEx). Blood glucose concentrations, insulin dosages, and hypo- or hyperglycemia events were controlled up to $24 \mathrm{~h}$ after exercise tests. Hypoglycemia was diagnosed with a BG concentration $<70 \mathrm{mg} / \mathrm{dL}$, hyperglycemia with fasting BG $>130 \mathrm{mg} / \mathrm{dL} .{ }^{4}$ Prior to and after the hypoxic exposure, as well as during both exercise protocols, pulse oxygen saturation $\left(\mathrm{SatO}_{2}\right.$, pulse oximeter), heart rate (HR, PE-3000 Sport-Tester; Polar Inc., Kempele, Finland) and systolic and diastolic blood pressure were measured (HEM-907XL; Omron Corporation, Kyoto, Japan).

\section{Biochemical analyses}

Blood samples were taken from the cubital vein in the morning at rest for the determination of $\mathrm{HbA}_{1 \mathrm{c}}$ (Ames DCA$2000^{\mathrm{TM}}$ Immunoassay Analyzer - normal range: 4.2-6.5\%), BG (Glucose 201+, HemoCue) and cytokine/hormone concentrations. The levels of HIF- $1 \alpha$, VEGF, and TGF- $\beta$ were measured by enzyme-linked immunosorbent assay ELISA kit (BlueGene Bitech Co., Ltd., Shanghai, China). Serum insulin (INS) and tumor necrosis factor alpha (TNF- $\alpha$ ) were measured by immunoassays (DIAsource ImmunoAssays, Ottignies-Louvain-la-Neuve, Belgium). Serum IGF-1 was determined using an immunoradiometric assay IRMA kit (IGF-1-RIACT Cisibo, Gif-sur-Yvette, France). Blood red blood cells (RBC), white blood cells (WBC), lymphocytes (LYM), monocytes (MON), absolute neutrophil count (ANC), and hemoglobin (HGB) level were measured (ABX MICROS 60, HORIBA, Montpellier, France). Blood lactate concentrations (LA) were determined using the Biosen C-line method (EKF Diagnostic $\mathrm{GmbH}$, Barleben, Germany); blood gases and acid-base balance were also analyzed (RapidLab 348; Bayer Diagnostics, Leverkusen, Germany). The D-max method was used to predict the anaerobic threshold based (LAT) on the recorded blood lactate levels. Data estimated in plasma after exercise was corrected for the after-exercise hematocrit change value. The obtained serum was aliquoted and frozen at $-80^{\circ} \mathrm{C}$ until assay.

\section{Statistical analysis}

All results are presented as mean \pm SD. The data was analyzed by a two-way ANOVA followed by the StudentNewman-Keuls test, when appropriate. The significance of the differences between groups was also assessed with 
the post hoc Bonferroni correction. All analyses were performed using Statistica v. 10 software package (StatSoft, Tulsa, USA). Statistical significance was set at $\mathrm{p}<0.05$.

\section{Results}

\section{Blood glucose, insulin dosage and diet}

Subjects with T1DM and CG were matched for age and they did not differ in body weight and composition (Table 1). Average calorie supply with diet, mean daily fat and protein intake on successive days of the experiment did not differ significantly between the CG and T1DM groups. The T1DM group demonstrated lower carbohydrate

Table 1. Subject characteristics (mean, SD)

\begin{tabular}{|l|c|c|}
\hline \multicolumn{1}{|c|}{ Variable } & $\begin{array}{c}\text { CG } \\
(\mathrm{n}=14)\end{array}$ & $\begin{array}{c}\text { T1DM } \\
(\mathrm{n}=14)\end{array}$ \\
\hline Age [years] & $24.0(5.2)$ & $30.4(9.7)$ \\
\hline Body height $[\mathrm{cm}]$ & $174.9(7.9)$ & $177.6(9.6)$ \\
\hline Body weight $[\mathrm{kg}]$ & $70.6(9.4)$ & $76.0(11.1)$ \\
\hline BMI [kg/m $\left.{ }^{2}\right]$ & $23.2(2.4)$ & $24.1(3.1)$ \\
\hline BFM [\%] & $17.1(5.9)$ & $18.3(8.2)$ \\
\hline FFM [kg] & $58.5(8.4)$ & $61.6(10.6)$ \\
\hline TBW [kg] & $43.0(6.2)$ & $45.3(7.8)$ \\
\hline WHR [cm] & $82.7(5.2)$ & $87.2(4.9)$ \\
\hline HbA ${ }_{1 c}[\mathrm{mmol} / \mathrm{mol}]$ & n.a. & $55.2(7.8)$ \\
\hline Duration of T1DM [years] & n.a. & $12.1(6.0)$ \\
\hline
\end{tabular}

BMI - body mass index; PBF - percentage body fat; BFM - body fat mass; FFM - fat-free body mass; TBW - total body water; WHR - waist-to-hip ratio; $\mathrm{HbA}_{1 c}$ - glycated hemoglobin; n.a. - not analyzed.

Table 2. Mean calorie supply with diet, mean daily fat, carbohydrate and protein intake on successive days of the experiment in CG and T1DM (mean, SD)

\begin{tabular}{|l|c|c|}
\multicolumn{1}{|c|}{ Variable } & $\begin{array}{c}\text { CG } \\
(\mathbf{n}=14)\end{array}$ & $\begin{array}{c}\text { T1DM } \\
(\mathbf{n}=14)\end{array}$ \\
\hline Calorie supply with diet $[\mathrm{kcal} / \mathrm{kg} /$ day $]$ & $35.7(9.4)$ & $31.1(11.9)$ \\
\hline Fat intake $[\mathrm{g} / \mathrm{kg} / \mathrm{day}]$ & $1.4(0.4)$ & $1.2(0.7)$ \\
\hline Carbohydrate intake $[\mathrm{g} / \mathrm{kg} /$ day $]$ & $4.4(1.7)$ & $3.6(1.5)^{\#}$ \\
\hline Protein intake $[\mathrm{g} / \mathrm{kg} / \mathrm{day}]$ & $1.7(0.5)$ & $1.5(0.8)$ \\
\hline
\end{tabular}

${ }^{*} \mathrm{p}<0.05$ significant differences between CG and T1DM.

Table 3. Fasting glycemia and insulin dosage on day 1 and day 2 of experiment in T1DM

\begin{tabular}{|l|c|c|}
\multicolumn{1}{|c|}{ Variable } & Nor & Hy \\
\hline Fasting glycemia day 1 [mg/dL] & $123.5(19.1)$ & $132.0(21.2)$ \\
\hline Fasting glycemia day 2 [mg/dL] & $115.5(44.5)$ & $116.0(17.0)$ \\
\hline Insulin day 1 [units/day] & $34.7(11.6)$ & $33.9(12.6)$ \\
\hline Insulin day 2 [units/day] & $35.9(13.6)$ & $35.9(11.6)$ \\
\hline
\end{tabular}

Fasting glycemia - blood glucose concentration after an overnight fast; insulin - insulin dosage; day 1 - the day of the Nor and Hy exercise; day 2 - the day following the Nor and Hy exercise tests. consumption compared to CG $(\mathrm{p}<0.05)$ (Table 2). The statistical analyses did not reveal any significant differences in fasting glycemia, nor in insulin dosage on the $1^{\text {st }}$ and $2^{\text {nd }}$ day in Nor and Hy conditions (Table 3).

\section{Normoxic and hypoxic rest}

Significantly lower $\mathrm{SatO}_{2}$ was observed after 40 min exposure to HyRest compared to NorRest (91.0 vs 96.0\%, respectively; $\mathrm{p}<0.05)$. Blood glucose (BG) concentrations were lower in CG compared to patients with T1DM at baseline and in response to resting hypoxia (Table 4). Forty-minute hypoxia did not induce significant changes in BG in T1DM. No significant effect of hypoxia was observed regarding WBC, RBC, HCT, and HBG concentrations (Table 5). The resting hypoxic intervention significantly altered HIF-1 $\alpha$ $(\mathrm{F}=5.1 ; \mathrm{p}<0.05)$. Significant increases of rest HIF-1 $\alpha$ level were observed in the T1DM group $(\mathrm{p}<0.001)$ and CG $(\mathrm{p}<0.05)$. Diabetes has a significant impact on resting HIF-1 $\alpha$ level $(\mathrm{p}<0.001)$ in comparison to healthy controls. Significantly lower TGF- $\beta_{\text {rest }}$ level $(p<0.05)$ in Hy compared to Nor conditions was observed in T1DM subjects (Table 6).

\section{Normoxic and hypoxic exercise test}

Peak oxygen consumption $\left(\mathrm{VO}_{2}\right)$, the criterion measure for aerobic capacity, showed good exercise tolerance in patients with T1DM (Table 4). A tendency toward increased $\mathrm{VO}_{2 \max }$ and significant higher minute pulmonary ventilation was observed in both groups in response to exercise and hypoxia. ANOVA showed significant interaction effects of the test conditions (NorEx vs HyEx) and group (T1DM vs CG) on $\mathrm{VE}_{\max }(\mathrm{F}=21.1, \mathrm{p}<0.001), \mathrm{HR}_{\max }$ $(\mathrm{F}=4.1 ; \mathrm{p}<0.05)$, and LA $(\mathrm{F}=6.4, \mathrm{p}<0.01)$. Post-hoc analysis confirmed higher values of $\mathrm{VE}_{\max }$ in $\mathrm{HyEx}$ than NorEx for both T1DM and CG ( $\mathrm{p}<0.01)$. Pre- and postexercise HR as well as SBP/DBP did not differ between both protocols $(\mathrm{p}>0.05)$.

No statistically significant effect of hypoxia on lactate threshold (LAT) was observed. However, the participants in both groups exhibited a tendency toward lower LAT in HyEx compared to NorEx. Moreover, significantly greater 15 min post-exercise LA level in HyEx compared to NorEx $(8.8 \pm 1.0$ vs $10.3 \pm 0.8 \mathrm{mmol} / \mathrm{L}$, respectively; $\mathrm{p}<0.05)$ was observed in T1DM subjects. Significantly lower $\mathrm{SatO}_{2}$ was observed after both exercise protocols $(\mathrm{p}<0.001)$ and postexercise $\mathrm{pO}_{2 \max }(\mathrm{p}<0.01)$ and $\mathrm{pCO}_{2 \max }(\mathrm{p}<0.01)$ (Table 4).

Two-way ANOVA revealed a significant effect of hypoxia and physical exercise on blood glucose concentrations $(\mathrm{F}=6.1 ; \mathrm{p}<0.01)$. In the T1DM group, lower glucose levels were observed in normobaric hypoxia compared to baseline and post-exercise levels in normoxia $(\mathrm{p}<0.05)$. Hypoxia and exercise (HyEx) had a significant impact on $\operatorname{HyEx}(\mathrm{F}=5.8 ; \mathrm{p}<0.01)$. Compared to baseline, NorEx and HyEx resulted in a significant decrease in BG ( $p<0.05$ vs $\mathrm{p}<0.001)$. Significantly lower BG levels were observed at 
Table 4. Cardiopulmonary indicators in hypoxia (Hy) and normoxia (Nor) for T1DM and CG

\begin{tabular}{|c|c|c|c|c|}
\hline \multirow{2}{*}{ Variable } & \multicolumn{2}{|c|}{ T1DM } & \multicolumn{2}{|c|}{ CG } \\
\hline & $\mathrm{Hy}$ & Nor & $\mathrm{Hy}$ & Nor \\
\hline $\mathrm{VO}_{2 \max }\left[\mathrm{mL} \cdot \mathrm{kg}^{-1} \cdot \mathrm{min}^{-1}\right]$ & $43.9(7.8)$ & $40.3(7.3)$ & $46.4(7.7)$ & $45.4(9.7)$ \\
\hline$P_{\max }[W]$ & $207.7(9.2)$ & $216.9(12.0)$ & $218.0(8.6)$ & $230.0(11.0)$ \\
\hline $\mathrm{LA}_{\max }[\mathrm{mmol} / \mathrm{L}]$ & $9.4(1.2)$ & $9.2(1.5)$ & $9.3(1.8)$ & $9.4(1.2)$ \\
\hline LAT [W] & $145.9(35.0)$ & $153.7(45.0)$ & $140.3(23.7)$ & $156.0(31.5)$ \\
\hline$V E_{\max }[\mathrm{L} / \mathrm{min}]$ & $121.0(26.0)$ & $101.6(24.0)^{* *}$ & $121.9(21.3)$ & $102.3(24.0)^{* *}$ \\
\hline$H R_{\text {pre }}[\mathrm{L} / \mathrm{min}]$ & $81.0(14.0)$ & $88.0(15.0)$ & $82.0(15.0)$ & $86.0(15.0)$ \\
\hline$H R_{\max }[\mathrm{L} / \mathrm{min}]$ & $177.0(10.0)$ & $178(12.0)$ & $181.0(12.0)$ & $183.0(11.0)$ \\
\hline $\mathrm{SBP}_{\text {pre }}[\mathrm{mm} \mathrm{Hg}]$ & $119.0(18.0)$ & $118.0(12.0)$ & $116.0(12.0)$ & $112.0(8.0)$ \\
\hline $\mathrm{SBP}_{\max }[\mathrm{mm} \mathrm{Hg}]$ & $150.0(9.0)$ & $163.0(13.0)$ & $149.0(12.0)$ & $145.0(13.0)$ \\
\hline $\mathrm{DBP}_{\text {pre }}[\mathrm{mm} \mathrm{Hg}]$ & $77.0(7.0)$ & $72.0(6.0)$ & $72.0(7.0)$ & $70.0(8.0)$ \\
\hline $\mathrm{DBP}_{\max }[\mathrm{mm} \mathrm{Hg}]$ & $76.0(8.0)$ & $72.0(6,0)$ & $72.0(7.0)$ & $74.0(7.0)$ \\
\hline $\mathrm{SatO}_{2 \max }[\%]$ & $90.8(4.1)$ & $97.0(3.1)^{* * *}$ & $92.0(0.8)$ & $96.8(1.0)^{* * *}$ \\
\hline $\mathrm{pO}_{2 \max }[\mathrm{mm} \mathrm{Hg}]$ & $62.8(1.8)$ & $86.5(1.6)^{* *}$ & $60.2(2.0)$ & $87.3(1.5)^{* *}$ \\
\hline $\mathrm{pCO}_{2 \max }[\mathrm{mm} \mathrm{Hg}]$ & $29.0(1.0)$ & $33.4(1.2)^{* *}$ & $29.1(0.9)$ & $33.1(1.0)^{* *}$ \\
\hline $\mathrm{BG}_{\text {pre }}[\mathrm{mg} / \mathrm{dL}]$ & $182(10.8)$ & $196.4(12.8)$ & $92.5(12.0)^{\# \# \#}$ & $92.9(16.6)^{\# \# \#}$ \\
\hline$B G_{\max }[\mathrm{mg} / \mathrm{dL}]$ & $141.3(9.2)$ & $170.4(11.3)^{* *}$ & $92.4(10.6)^{\# \# \#}$ & $91.1(15.7)^{\# \# \#}$ \\
\hline
\end{tabular}

$\mathrm{VO}_{2}$ - oxygen consumption; P - power; LA - blood lactate concentration; LAT - lactate threshold; VE - lung minute ventilation; HR - heart rate;

$\mathrm{SBD}$ - systolic blood pressure; DBD - diastolic blood pressure; $\mathrm{SatO}_{2}$ - oxyhemoglobin saturation; $\mathrm{pO}_{2}$ - partial pressure of oxygen in blood; $\mathrm{pCO} \mathrm{O}_{2}$ - partial pressure of carbon dioxide in blood; BG - blood glucose; pre - measured at rest before exercise; max - measured in the last minute of the exercise test; ${ }^{*} p<0.05$ and ${ }^{* *} p<0.001$ indicate statistically significant differences between Nor and Hy conditions; ${ }^{\# \# \# ~}<0.001$ indicates statistically significant differences between CG and T1DM.

Table 5. Blood response measures following hypoxic (Hy) and normoxic (Nor) conditions

\begin{tabular}{|c|c|c|c|c|c|c|c|}
\hline \multirow{2}{*}{\multicolumn{2}{|c|}{ Variable }} & \multicolumn{3}{|c|}{ TIDM } & \multicolumn{3}{|c|}{ CG } \\
\hline & & Rest & PreEx & $\mathrm{Ex}_{\max }$ & Rest & PreEx & $\mathrm{Ex}_{\max }$ \\
\hline \multirow{2}{*}{$\begin{array}{l}\text { LYM } \\
{\left[10^{9} / \mathrm{L}\right]}\end{array}$} & Hy & $2.1(0.7)$ & $2.2(0.8)$ & $4.2(0.3)^{* *}$ & $2.1(0.4)$ & $2.0(0.6)$ & $4.1(0.5)^{* * *}$ \\
\hline & Nor & $2.1(0.6)$ & $2.1(0.6)$ & $3.2(0.3)^{*}$ & $2.2(0.5)$ & $1.9(0.4)$ & $3.4(0.3)^{* * *}$ \\
\hline \multirow{2}{*}{$\begin{array}{l}\text { ANC } \\
{\left[10^{9} / L\right]}\end{array}$} & Hy & $2.1(1.6)$ & $2.3(1.0)$ & $3.2(0.5)$ & $3.2(1.2)$ & $3.4(1.3)$ & $4.8(0.5)^{* * \#}$ \\
\hline & Nor & $2.4(0.8)$ & $2.0(0.5)$ & $2.6(0.9)$ & $3.1(1.1)$ & $3.8(1.1)$ & $5.2(0.8)^{* * \#}$ \\
\hline \multirow{2}{*}{$\begin{array}{l}\mathrm{MON} \\
{\left[10^{9} / \mathrm{L}\right]}\end{array}$} & Hy & $0.7(0.3)$ & $0.7(0.3)$ & $0.7(0.1)$ & $0.5(0.3)$ & $0.4(0.2)$ & $0.9(0.1)^{* * *}$ \\
\hline & Nor & $0.6(0.2)$ & $0.5(0.2)$ & $0.6(0.1)$ & $0.3(0.1)$ & $0.3(0.2)$ & $0.5(0.1)^{*}$ \\
\hline \multirow{2}{*}{$\begin{array}{l}\text { WBC } \\
{\left[10^{9} / \mathrm{L}\right]}\end{array}$} & Hyp & $5.8(2.3)$ & $4.9(1.9)$ & $8.7(0.9)^{* * *}$ & $5.0(1.1)$ & $5.8(1.6)$ & $9.8(1.2)^{* * * *}$ \\
\hline & Nor & $5.0(1.9)$ & $4.7(1.6)$ & $7.1(1.1)$ & $4.7(1.2)$ & $6.0(2.0)$ & $9.0(1.1)^{* * *}$ \\
\hline \multirow{2}{*}{$\begin{array}{l}\mathrm{HGB} \\
{[\mathrm{g} / \mathrm{L}]}\end{array}$} & Hy & $14.8(0.9)$ & $15.1(0.9)$ & $15.9(0.5)$ & $15.5(0.6)$ & $15.9(0.9)$ & $16.1(0.8)$ \\
\hline & Nor & $15.1(0.9)$ & $15.1(0.9)$ & $16.0(0.8)$ & 16.1 (0.9)\# & $15.3(1.1)$ & $15.6(0.6)$ \\
\hline \multirow{2}{*}{$\begin{array}{l}\mathrm{HCT} \\
{[\mathrm{L} / \mathrm{L}]}\end{array}$} & Hy & 43.7 (3.8) & $44.2(2.0)$ & $46.8(1.7)$ & $47.2(1.9)$ & $46.0(1.9)$ & $47.6(2.2)$ \\
\hline & Nor & $44.2(2.0)$ & $43.7(3.8)$ & $48.7(2.9)$ & $45.9(2.1)$ & $45.2(2.0)$ & $47.9(2.2)$ \\
\hline \multirow{2}{*}{$\begin{array}{l}\text { RBC } \\
{\left[10^{12} / L\right]}\end{array}$} & Hy & $4.9(0.2)$ & $4.9(0.5)$ & $5.3(0.2)$ & $5.4(0.2) \#$ & $5.3(0.4)$ & $5.5(0.2)$ \\
\hline & Nor & $4.9(0.4)$ & $4.9(0.2)$ & $5.2(0.3)$ & 5.5 (0.3)\# & $5.5(0.2)^{\#}$ & $5.5(0.3)$ \\
\hline
\end{tabular}

LYM - lymphocytes; ANC - absolute neutrophil count; MON - monocytes; WBC - white blood cells; HGB - hemoglobin; HCT - hematocrit; RBC - red blood cells; rest - measured at rest after 40 min of Nor and Hy; preEx - measured at rest before exercise; Ex max - measured in the last minute of the exercise tests. ${ }^{*} p<0.05,{ }^{* *} p<0.01,{ }^{* * *} p<0.001$ indicate statistically significant differences between max and preEx value; ${ }^{*} p<0.05$ indicates statistically significant differences between CG and T1DM group.

maximal exercise intensity $(\mathrm{p}<0.01)$ and in response to a $15-$ min and 24-h recovery period after Hy Ex $(\mathrm{p}<0.05)$ compared to NorEx (Fig. 1). A tendency toward lower fasting glycemia on day 2 after graded exercise compared to day 1 was observed (Table 3). The analysis of the incidence of hyper- or hypoglycemia indicated that hyperglycemia episodes were more frequent in patients after NorEx compared to HyEx ( $30 \%$ and $20 \%$, respectively).

Significant increases in WBC $(\mathrm{p}<0.01)$ and LYM count $(\mathrm{p}<0.01)$ were observed in response to HyEx compared 
Table 6. Angiogenic factors in hypoxia (Hy) and normoxia (Nor) for T1DM and CG

\begin{tabular}{|c|c|c|c|c|}
\hline \multirow{2}{*}{ Variable } & \multicolumn{2}{|c|}{ T1DM } & \multicolumn{2}{|c|}{ CG } \\
\hline & $\mathrm{Hy}$ & Nor & $\mathrm{Hy}$ & Nor \\
\hline HIF-1a $a_{\text {rest }}[\mathrm{ng} / \mathrm{mL}]$ & $217.5(18.0)$ & $36.1(22.0)^{* * *}$ & $32.2(5.2)^{\# \# \# ~}$ & $20.1(10.7)^{* \#}$ \\
\hline $\mathrm{HIF}-1 \mathrm{a}_{\max }[\mathrm{ng} / \mathrm{mL}]$ & $163.0(48.6)$ & $48.7(21.6)^{* *}$ & $38.0(16.4)^{\# \# \#}$ & $23.6(8.9)^{* \#}$ \\
\hline$V E G F_{\text {rest }}[\mathrm{pg} / \mathrm{mL}]$ & $15.0(8.5)$ & $10.0(8.7)$ & $11.4(6.5)$ & $22.6(15.8)$ \\
\hline$V_{E G F_{\max }}[\mathrm{pg} / \mathrm{mL}]$ & $10.3(8.1)$ & $23.7(12.3)^{*}$ & $14.4(8.1)$ & $17.4(11.3)$ \\
\hline TGF- $\beta_{\text {rest }}[\mathrm{pg} / \mathrm{mL}]$ & $113.0(38.8)$ & $141.0(28.7)^{*}$ & $121.5(45.5)$ & $131.0(18.8)$ \\
\hline TGF- $\beta_{\max }[\mathrm{pg} / \mathrm{mL}]$ & $144.7(34.2)$ & $152.0(32.1)$ & $158.0(34.8)$ & $143.0(42.0)$ \\
\hline TNF-a $a_{\text {rest }}[\mathrm{pg} / \mathrm{mL}]$ & $61.6(23.5)$ & $35.6(16.9)$ & $50.2(30.0)$ & $35.1(10.4)$ \\
\hline TNF-a $a_{\max }[\mathrm{pg} / \mathrm{mL}]$ & $43.6(14.0)$ & $39.4(14.4)$ & $43.2(14.3)$ & $34.3(7.7)$ \\
\hline $\mid G F-1_{\text {rest }}[\mathrm{ng} / \mathrm{mL}]$ & $212.0(80.0)$ & $214.0(86.0)$ & $486.2(75.0)^{\# \#}$ & $474.0(60.0)^{\# \#}$ \\
\hline $\mid G F-1_{\max }[\mathrm{ng} / \mathrm{mL}]$ & $233.0(83.0)$ & $215.0(98.0)$ & $573.3(58.3)^{\# \#}$ & $550.0(99.0)^{\#}$ \\
\hline $\mathrm{INS}_{\text {rest }}[\mathrm{ng} / \mathrm{mL}]$ & $7.4(4.0)$ & $5.1(2.0)$ & $14.2(5.3)$ & $14.2(5.3)$ \\
\hline $\mathbb{N N S}_{\max }[\mathrm{ng} / \mathrm{mL}]$ & $7.1(2.9)$ & $4.4(0.6)$ & $21.7(12.0)$ & $15.0(8.2)$ \\
\hline
\end{tabular}

${ }^{*} p<0.05$ and ${ }^{* *} p<0.001$ indicate statistically significant differences between normoxic and hypoxic conditions; $\# p<0.05 ; \# p<0.01 ; \#$;\#\# $p<0.001$ indicate statistically significant differences between CG and T1DM.

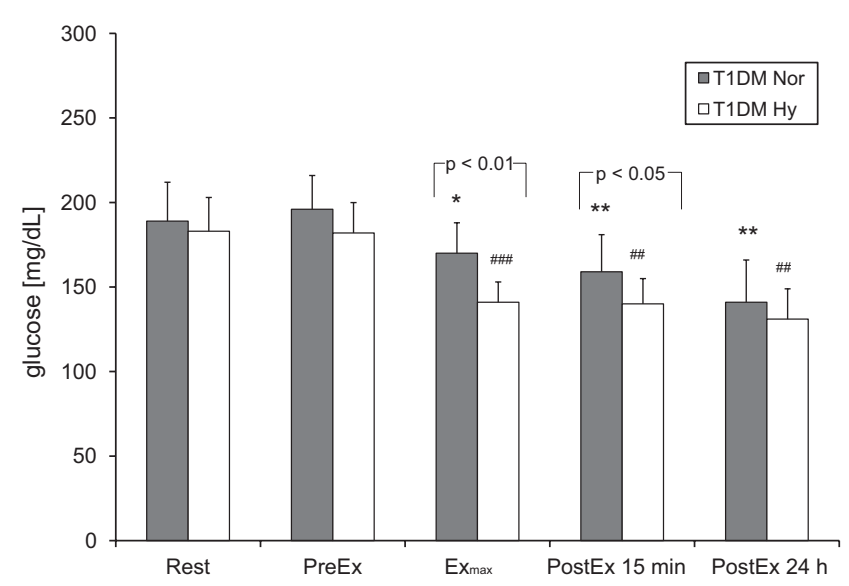

Fig. 1. Blood glucose concentrations at rest, pre-exercise (PreEx), at maximal exercise intensity $\left(\mathrm{Ex}_{\max }\right)$, and in response to 15-min (PostEx $15 \mathrm{~min}$ ) and 24-h recovery (PostEx $24 \mathrm{~h}$ ) in normoxia (Nor) and hypoxia $(\mathrm{Hy})$ in patients with diabetes (T1DM)

${ }^{*} p<0.05,{ }^{* *} p<0.01,{ }^{* * *} p<0.001$ indicate statistically significant differences between preEx and post exercise value in Nor; $\#$ \# $<0.01$, $\# \# p<0.001$ indicate statistically significant differences between preEx and post exercise value in $\mathrm{Hy}$.

to preEx count in T1DM. Exercise under Hy and Nor conditions significantly increased LYM ( $<<0.001)$, ANC ( $\mathrm{p}<0.01)$, MON $(\mathrm{p}<0.001)$ and WBC counts $(\mathrm{p}<0.001)$ in CG. ANC was significantly higher at maximal exercise intensity (Hy and Nor) in CG compared to T1DM ( $\mathrm{p}<0.05)$. T1DM participants exhibited a significant reduction in RBC ( $<$ 0.05) (Table 5).

The HyEx had a significant effect on serum HIF-1 $\alpha$ concentrations $(F=5.1 ; p<0.05)$. Significant increases of postexercise HIF-1 $\alpha$ (HyEx; $\mathrm{p}<0.01)$ were observed compared to Nor in T1DM and CG ( $<<0.05)$. Diabetes has a significant impact on post-exercise HIF- $1 \alpha$ level ( $p<0.001)$.
A tendency toward higher VEGF level was observed after NorEx compared to HyEx ( $\mathrm{p}=0.05)$ and a non-significant decrease was seen in response to HyEx compared to HyRest in T1DM. Hypoxia significantly decreased baseline TGF- $\beta$ $(\mathrm{p}<0.05)$ and had a significant effect on TNF- $\alpha$ level $(\mathrm{F}=4.9 ; \mathrm{p}<0.05)$. No significant effects of hypoxia were observed regarding pre- and post-exercise INS and IGF-1 levels. In both conditions, significantly lower serum IGF-1 concentrations were observed in T1DM $(p<0.01)$. There was a tendency toward higher IGF-1 levels after exercise in HyEx compared to NorEx in both groups (Table 6).

\section{Discussion}

\section{Effects of high intensity exercise in normoxia and normobaric hypoxia}

According to scientific reports, the primary aim of diabetes treatment is to achieve as stable glycemic control as possible to prevent the long term complications. ${ }^{1-4}$ The present data confirmed that hypoxia combined with physical activity have been shown to induce beneficial effects on glycemic control, the cardiovascular system and exercise tolerance. Glucose control was improved after lowand moderate-intensity exercise, but also in response to high-intensity exercise combined with hypoxia. ${ }^{3,8,10,15,16,23}$

Mackenzie et al. (in 2011 and 2012) have recently demonstrated that combining physical exercise with hypoxia has an additive effect on glucose utilization in people with T2DM. ${ }^{15,23}$ The authors also noted that aerobic as well as anaerobic exercise improve glycemia control, however, to a different extent.

The major findings of our study are that a single bout of graded exercise reduces glycemia in T1DM. The greatest 
and a safe decline in blood glucose concentration was stated after exercise combined with exposure to hypoxia. In this study, intense aerobic exercise in hypoxic conditions decreases the hyperglycemic episodes compared to NorEx and allows more effective control of post-exercise glucose homeostasis. In patients with T1DM in normoxic conditions, a high intensity exercise-induced increase of BG concentration is followed by hypoglycemia hours after completing the exercise. Indeed, in the present study, episodes of hyperglycemia were mainly observed in response to NorEx. Blood glucose concentration in the postexercise period could have been influenced by patients' diet and insulin administration. However, on day 2, patients in fact applied the same doses of insulin compared to day 1. Therefore, the differences of hyperglycemia mainly depended on the exercise test conditions. Hypoxia and muscle contractions reduce elevated blood glucose levels more effectively, improve short-term glycemic control and may be important in preventing post-exercise hyperglycemic events.

It is well established that constant administration of various insulin preparations, proper diet and regular physical activity are essential to optimize glycemia control and minimize potential diabetes complications. ${ }^{4,8}$ Recent investigations also indicate that paracrine mechanisms, observed during hypoxia exposure in isolated insulin-resistant muscle tissue and in humans, may exert alternative or parallel actions. ${ }^{21,24}$ Mackenzie et al. have shown lower BG concentrations during hypoxia exposure associated with an increase in INS sensitivity in T2DM. ${ }^{15,23}$ In this study, the markedly lower BG level in T1DM patients after exercising in hypoxic conditions may depend on higher absorption of insulin in hypoxia due to vasodilatation with increased endothelium-dependent dilator capacity, higher production of endothelial nitric oxide (NO), and eNOS expression. The possible mechanism might consist of up-regulation of the body's glycolytic energy pathways to compensate for hypoxia-induced reduction in mitochondrial respiration. In our study, we could only assume that a significant increase in post-exercise LA level and lower LAT in HyEx might suggest higher stimulation of anaerobic glycolytic pathways under hypoxic conditions. These observations seem to confirm the abovementioned significant role of anaerobic metabolism in adapting to hypoxia and the possible use of glycolytic processes in patients with T1DM.

Data concerning the effects of exercise and exposure to hypoxic conditions on aerobic performance and health condition is still sparse. Most studies report beneficial effects of this type of exercise compared to exercise in normoxia in healthy individuals. ${ }^{25,26}$ However, there is not enough data on the effects of hypoxia with and without exercise on the condition of the cardiovascular and immune systems of people with diabetes. ${ }^{5,6,10}$

\section{Angiogenic factors in response to normobaric hypoxia}

It has been previously documented that diabetes is associated with angiopathy, the latter being possibly aggravated by angiogenesis. ${ }^{11,13}$ An important result presented in this study is the significant effect of diabetes on the level of proangiogenic factors. Patients with diabetes had significantly higher baseline HIF-1 $\alpha$ levels and lower serum IGF-1 concentrations compared to healthy control. There were no differences in baseline VEGF and TNF- $\alpha$ between T1DM and CG. The higher HIF-1 $\alpha$ level and the lower serum IGF-1 concentrations observed in T1DM compared to healthy subjects might suggest that these factors may modify angiogenesis processes.

The major finding of the study is that $40 \mathrm{~min}$ exposure to hypoxia significantly decreased TGF- $\beta$ concentrations in patients with T1DM. This factor has anti-inflammatory action, so if hypoxia in T1DM lowers TGF- $\beta$ levels, it could suggest that hypoxia lowers the anti-inflammatory potential of these patients. However, the tendency toward higher TGF- $\beta$ and lower HIF- $1 \alpha$, VEGF and TNF- $\alpha$ observed in response to exercise after hypoxia compared to HyRest and lower VEGF after HyEx compared to NorEx may suggest the beneficial effects of exercise in hypoxia on the regulation of pro-angiogenic pathways and possible stabilization of angiogenesis in physically active patients.

According to Sanchez-Elsner et al., HIF-1 $\alpha$ stimulates the secretion of TGF- $\beta .{ }^{27}$ Interestingly, TGF- $\beta$ stimulates angiogenesis by inducing apoptosis of endothelial cells. It has been shown that elevated glycemia activates inflammatory cells to the production of this factor. Excessive secretion and activity of TGF- $\beta$ was confirmed in diabetic nephropathy and retinopathy, and also in other pathological conditions. ${ }^{28}$ In the present study, baseline levels of TGF- $\beta$ were lower in Hy conditions in the T1DM group. Since evaluation of TGF- $\beta$ is a valuable tool for the diagnosis of diabetic complications, the study findings could suggest a beneficial effect of hypoxia $\left(\mathrm{FIO}_{2}=15.2 \%\right)$ and exercise in the prevention of these disorders. No significant differences in post-exercise TGF- $\beta$ concentrations in the T1DM group compared to healthy subjects allows us to assume that this factor could not contribute to cardiovascular complications in response to high intensity exercise.

In our study, we also analyzed blood markers for immune function in people with diabetes and healthy individuals. A more pronounced increase in circulating monocytes and neutrophils as well as higher LYM and WBC counts immediately after exercise in response to HyEx and NorEx in CG seemed to suggest greater immune activation - probably in response to higher sympathetic activity compared to T1DM subjects. Type 1 diabetes mellitus is characterized by beta cell destruction caused by an autoimmune process, usually leading to absolute insulin deficiency. ${ }^{1}$ Under physiological conditions, there is a balance between pathogenic $\mathrm{T}$ cells that mediate disease and regulatory cells 
that control autoimmunity. However, an imbalance in the activity between the pathogenic actions of auto-reactive effector T cells (Teffs) and a second T cell subtype, known as regulatory $\mathrm{T}$ cells (Tregs), may be crucial in the breakdown of peripheral tolerance, leading to the development of T1DM. ${ }^{29}$

According to our study, hypoxia and exercise did not increase cytokine concentrations. Moreover, the results of leucocyte measurements indicated that exercise under hypoxic conditions may induce lower immune response in T1DM compared to CG and allows a hypothesis that exposure to $\mathrm{HyEx}\left(\mathrm{FIO}_{2}=15.2 \%\right)$ is not a factor leading to an increased number of diabetes complications. It is worth pointing out that our participants with T1DM had baseline $\mathrm{HbA}_{1 \mathrm{C}}$ levels slightly over the reference range, suggesting proper long-term glycemia control and the effects of diabetes on the immune system might not be so spectacular.

Since multiple signaling pathways may be responsible for preventing T1DM complications, we also analyzed exercise-induced serum IGF-1 responses. The previous data demonstrated that administration of IGF-1 and/or a complex of IGF-1/IGFBP-3 effectively enhanced $\beta$-cell resistance to cytokine-induced cytotoxicity and might prove an efficacious therapy for T1DM complication prevention. ${ }^{30}$ On the other hand, the results of in vitro and in vivo studies have demonstrated that IGF-1 activates the phosphatidyl inositol (PI), 3-kinase/Akt and mitogen-activated protein kinase (MAPK) signaling pathways and induces the growth of myocytes. The pathways play a significant role in the induction of muscle hypertrophy and an increase in IGF-1 secretion in response to hypoxic conditions accompanied by reduced glycemia. This might constitute an important mechanism in the prevention of skeletal muscle hypotonia and cardiomyocyte dysfunction in T1DM patients. Our results allow us to assume that HyEx might constitute an important factor leading to IGF-1 elevation. As we did not assess differences in the levels of other hormones, we could only hypothesize that the exercise- and hypoxia-induced increase in IGF-1 availability in tissues might act as an important protective factor against the aforementioned diabetes complications.

Another important result presented in our study is the significant effect of Hy on the levels of proangiogenic factors. Diabetes has a significant impact on both resting and post-exercise HIF-1 $\alpha$ levels, but not on the main angiogenic factor, i.e., VEGF. In this study, lower VEGF levels were observed after HyEx compared to NorEx. Importantly, lower serum concentrations of TGF- $\beta$ and a tendency toward higher IGF-1 were seen in response to exercise in hypoxia compared to normoxia in T1DM. However, no significant effect of exogenous insulin dose was observed in serum INS concentrations in Hy compared to NorRest and NorEx in both T1DM groups.

Diabetes mellitus is a generally accepted risk factor for vascular dysfunction, contributing to the development of serious complications. ${ }^{11,13,20}$ The coexistence of enhanced angiogenesis, such as in proliferative retinopathy or atherosclerotic plaque angiogenesis, and impaired neovascularization in diabetes is defined as "the angiogenic paradox". ${ }^{31}$ Diabetes related vascular complications can be caused by micro- and macroangiopathy depending on the increase in glycemia, inflammatory and oxidative stress, and is associated with deregulation of cellular and tissue response to ischemia. VEGF is a potent angiogenic factor. It stimulates the proliferation and differentiation of endothelial cells, prevents apoptosis of muscle and endothelial cells, and regulates vasodilatation. VEGF expression has been evidenced to increase under hypoxic conditions largely due to HIF- $1 \alpha \cdot{ }^{19,20}$ Hence, HIF- $1 \alpha$ mediates VEGF expression and stimulates inflammatory cells to secrete a direct stimulator of angiogenesis (TGF- $\beta$, TNF- $\alpha$ ), which has a pro- and antiangiogenic effect. The results of several studies on HIF-1 $\alpha$ expression in diabetes show both its inhibition and enhancement. Xiao et al. emphasized the stimulating effect of high BG on HIF- $1 \alpha$ synthesis and transcriptional activity in human epithelial cells. ${ }^{32}$ Thangarajah et al. reached the opposite conclusions, suggesting an inhibiting effect of hyperglycemia on HIF-1 $\alpha$ secretion in diabetes. ${ }^{19}$ The results of the present study revealed significantly higher baseline levels of HIF-1 $\alpha$ in T1DM subjects associated with hyperglycemia. Hypoxia increased the concentration of HIF- $1 \alpha$ probably in response to the effects of inhibition of its degradation. It could be suggested that hypoxia is much more challenging to patients with diabetes than to healthy subjects. Higher baseline HIF- $1 \alpha$ concentration during a hypoxic state could lead to the conclusion that hypoxia is not as beneficial for T1DM patients as for healthy individuals. However, it might be hypothesized that reduced hyperglycemia in response to hypoxia with exercise contributes to lower local stimulation of endothelial cells to express HIF- $1 \alpha$ and VEGF. Poor glycemic control causes higher serum HIF-1 $\alpha$ concentration and could be associated with marked serum VEGF increase. Hence, the improvement of glycemic control with the significant reduction in proangiogenic factors observed in our study in response to hypoxia and exercise helps decrease the risk of severe microvascular complications in T1DM. A revision of the recent findings published in the literature regarding the angiogenic paradox will be performed. Apparently, endothelial dysfunction, as well as molecules such as VEGF and HIF-1 $\alpha$, play a major role in vascular complications. In addition, the monocytes/ macrophages are important in endothelium activation for arteriogenesis and its arteriogenic response is reduced, leading to impaired collateral artery growth. Moreover, the molecular mechanisms involved will be addressed, including abnormalities in growth factor, cytokines and metabolic derangements. ${ }^{33}$ 


\section{Conclusions}

In summary, our results show that short-time hypoxia combined with graded exercise increases cardiorespiratory adaptation to exercise and allows more effective control of glucose homeostasis in patients with type 1 diabetes. The pattern of observed changes in pro- to anti-angiogenic factors suggests that hypoxia may provide benefits in the prevention and management of diabetes complications, even in patients participating in high intensity physical training. Whether this therapy might result in a long-term clinical benefit needs further investigation.

\section{Limitations}

Intensive insulin therapy impedes the interpretation of the effects of different study protocols on glycemic control in the group of patients. In addition, the concentrations of angiogenic and inflammatory factors in blood serum were assessed only immediately after exercise, which limits the ability to draw conclusions about the long-term therapeutic effects.

\section{References}

1. American Diabetes Association. Standards of medical care in diabetes - 2010: Current criteria for the diagnosis of diabetes. Diabetes Care. 2010;33(1):4-10.

2. Hayes $\mathrm{Ch}$, Kriska A. Role of physical activity in diabetes management and prevention. J Am Diet Assoc. 2008;108(1):19-23. doi:10.1016/j. jada.2008.01.016

3. Carral F, Gutiérrez JV, Ayala MD, García G, Aguilar M. Intense physical activity is associated with better metabolic control in patients with type 1 diabetes. Diabetes Res Clin Pract. 2013;101(1):45-49. doi:10. 1016/j.diabres.2013.04.006

4. American Diabetes Association. Standards of medical care in diabetes - 2015. Diabetes Care. 2015;38(1):10-38.

5. Pavan $P$, Sarto $P$, Merlo L, et al. Metabolic and cardiovascular parameters in type 1 diabetes at extreme altitude. Med Sci Sports Exerc. 2004;36(8):1283-1289.

6. Kalson NS, Davies AJ, Stokes S, et al. Climbers with diabetes do well on Mount Kilimanjaro. Diabet Med. 2007;24(12):1496.

7. Lehnen AM, Angelis KD, Markoski MM, Schaan BDA. Changes in the GLUT4 expression by acute exercise, exercise training and detraining in experimental models. J Diabetes Metab. 2012;10:002. doi:10. 4172/2155-6156

8. D'hooge R, Hellinckx T, Calders P. Influence of combined aerobic and resistance training on metabolic control, cardiovascular fitness and quality of life in adolescents with type 1 diabetes: A randomized controlled trial. Clin Rehabil. 2011;25(4):349-359. doi:10.1177/ 0269215510386254

9. Jensen L, Bangsbo J, Hellsten Y. Effect of high intensity training on capillarization and presence of angiogenic factors in human skeletal muscle. J Physiol. 2004;557:571-582.

10. Schobersberger W, Schmid P, Lechleitner M, et al. Austrian Moderate Altitude Study 2000 (AMAS). The effects of moderate altitude $(1,700 \mathrm{~m})$ on cardiovascular and metabolic variables in patients with metabolic syndrome. Eur J Appl Physiol. 2003;88:506-514.

11. Silvestre JS, Lévy BI. Molecular basis of angiopathy in diabetes mellitus. Circ Res. 2006;98:4-6.

12. Di Marzio D, Mohn A, Mokini Z, Giannini C, Chiarelli F. Macroangiopathy in adults and children with diabetes: From molecular mechanisms to vascular damage (part 1). Hormone Metab Res. 2006;38(11):691-705

13. Chiarelli F, Spagnoli A, Basciani F, et al. Vascular endothelial growth factor (VEGF) in children, adolescents and young adults with type 1 diabetes mellitus: Relation to glycaemia control and microvascular complications. Diabetic Med. 2000;17(9):650-656.

14. Wee J, Climstein M. Hypoxic training: Clinical benefits on cardiometabolic risk factors. J Sci Med Sport. 2015;18(1):56-61. doi:10.1016/j. jsams.2013.10.247

15. Mackenzie R, Maxwell N, Castle P, Brickley G, Watt P. Acute hypoxia and exercise improve insulin sensitivity $\left(\mathrm{S}(\mathrm{I})\left(2^{*}\right)\right.$ in individuals with type 2 diabetes. Diabetes/Metab Res Rev. 2011;27(1):94-101. doi:10. 1002/dmrr.1156

16. Kivelä R, Silvennoinen M, Touvra AM, Lehti TM, Kainulainen $H$, Vihko V. Effects of experimental type 1 diabetes and exercise training on angiogenic gene expression and capillarization in skeletal muscle. FASEB J. 2006;20(9):1570-1572.

17. Martin A, Komada MR, Sane DC. Abnormal angiogenesis in diabetes mellitus. Med Res Rev. 2003;23(2):117-145.

18. Mackenzie R, Elliott B, Maxwell N, Brickley G, Watt P. The effect of hypoxia and work intensity on insulin resistance in type 2 diabetes. J Clin Endocrinol Metab. 2012;97(1):155-162. doi:10.1210/jc.2011-1843

19. Thangarajah $\mathrm{H}$, Vial IN, Grogan $\mathrm{RH}$, et al. HIF-1alpha dysfunction in diabetes. Cell Cycle. 2010;9(1):75-79.

20. Thangarajah $\mathrm{H}, \mathrm{Yao} \mathrm{D}, \mathrm{Chang} \mathrm{El}$, et al. The molecular basis for impaired hypoxia-induced VEGF expression in diabetic tissues. Proc Natl Acad Sci USA. 2009:106(32):13505-13510. doi:10.1073/pnas.0906670106

21. Azevedo JL Jr, Carey JO, Pories WJ, Morris PG, Dohm GL. Hypoxia stimulates glucose transport in insulin-resistant human skeletal muscle. Diabetes. 1995;44(6):695-698.

22. Chen $\mathrm{CH}$, Liu YF, Lee SD, et al. Altitude hypoxia increases glucose uptake in human heart. High Alt Med Biol. 2009;10:83-86. doi:10.1089/ ham.2008.1064

23. Mackenzie R, Maxwell N, Castle P, Elliott B, Brickley G, Watt P. Intermittent exercise with and without hypoxia improves insulin sensitivity in individuals with type 2 diabetes. J Clin Endocrinol Metab. 2012;97(4):546-555. doi:10.1210/jc.2011-2829

24. Castillo O, Woolcott OO, Gonzales E, et al. Residents at high altitude show a lower glucose profile than sea-level residents throughout 12-hour blood continuous monitoring. High Alt Med Biol. 2007;8: 307-311.

25. Lundby C, Calbet JAL, Robach P. The response of human skeletal muscle tissue to hypoxia. Cell Mol Life Sci. 2009;66:3615-3623. doi:10.1007/ s00018-009-0146-8

26. Millet GP, Roels B, Schmitt L, Woorons X, Richalet JP. Combining hypoxic methods for peak performance. Sports Med. 2010;40(1):1-25.

27. Sánchez-Elsner T, Botella LM, Velasco B, Corbí A, Attisano L, Bernabéu C. Synergistic cooperation between hypoxia and transforming growth factor-beta pathways on human vascular endothelial growth factor gene expression. J Biol Chem. 2001;276(42):38527-38535.

28. Shaker YM, Hanan A, Soliman HA, et al. Serum and urinary transforming growth factor beta 1 as biochemical markers in diabetic nephropathy patients. Beni-Suef Univ J Basic App/Sci. 2014;3(1):16-23.

29. Cabrera SM, Rigby MR, Mirmira RG. Targeting regulatory T cells in the treatment of type 1 diabetes mellitus. Curr Mol Med. 2012:12(10); $1261-1272$.

30. Chen W, Salojin KV, Mi QS, Meagher TC, Zucker P, Delovitch TL. Insulin-like growth factor (IGF)-I/IGF-binding protein 3 complex: Therapeutic efficacy and mechanism of protection against type 1 diabetes. Endocrinology. 2004;145:627-638.

31. Simons M. Angiogenesis, arteriogenesis, and diabetes: Paradigm reassessed? J Am Coll Cardiol. 2005;46:835-837.

32. Xiao H, Gu Z, Wang G, Zhao T. The possible mechanisms underlying the impairment of HIF-1a pathway signaling in hyperglycemia and the beneficial effects of certain therapies. Int J Med Sci. 2013;10(10):1414-1421. doi:10.7150/ijms.5630

33. Kuehl MN, Rodriguez $\mathrm{H}$, Burkhard BR, Alman AC. Tumor necrosis factor-a, matrix-metalloproteinases 8 and 9 levels in the saliva are associated with increased hemoglobin a1c in type 1 diabetes subjects. PLoS One. 2015;10(4):e0125320. doi:10.1371/journal.pone. 0125320 